\title{
RARE-EARTH EXCITATION MECHANISM IN WIDE BAND GAP II-VI COMPOUNDS
}

\author{
K. Karpińska, K. ŚWiątek, M. Godlewski \\ Institute of Physics, Polish Academy of Sciences \\ Al. Lotników 32/46, 02-668. Warszawa, Poland
}

L. NIINISTö

Laboratory of Inorganic and Analytical Chemistry, Helsinki University of Technology 02150 Espoo, Finland

\section{AND M. LESKEL $\ddot{A}$}

Department of Chemistry, University of Helsinki, 00100 Helsinki, Finland

The excitation mechanism of rare-earth emission in $\mathrm{Eu}$ and Ce doped $\mathrm{CaS}$ and $\mathrm{SrS}$ is studied. It is proposed that the Eu and probably also Ce emission is induced by the photoionization transition of the rare-earth ion, which is followed by the carrier trapping via the excited state of the ion. At increased temperatures the efficiency of excitation is reduced. We explain this effect by the carrier emission from the excited core state of the rare-earth ion to the continuum of the conduction (valence) band states. It is also suggested that the charge transfer state of the rare-earth ion may act as the intermediate state in the carrier trapping.

PACS numbers: 71.55.Gs, 76.30.Kg, 78.55.Et

\section{Introduction}

Rare-earth (RE) activated wide band gap $\mathrm{CaS}$ and SrS belong to the most perspective candidates for producing efficient multicolor thin film electroluminescence (EL) displays [1]. Even though there are still problems with their chemical instability and hydroscopic nature, $\mathrm{CaS}$ and $\mathrm{SrS}$ doped with Eu and Ce can be applied for red (CaS:Eu) and blue (SrS:Ce) EL displays [2]. The main hindrance for obtaining the high EL efficiency from such displays was low excitation efficiency. This limitation can be avoided if the impact excitation of the RE ion is replaced by the impact ionization process [3]. This idea was verified in our recent studies of $\mathrm{Yb}$ doped $\mathrm{ZnS}$ and Eu doped $\mathrm{ZnS}$ and $\mathrm{CaS}$ [4-8]. It was shown that the efficient $\mathrm{Eu}^{2+}$ emission can be induced by the Eu ionization. This was explained by trapping of the ionized carrier via the $4 f^{6} 5 d^{1}$ excited state of the ion $\left(\mathrm{Eu}^{3+}+\mathrm{e} \rightarrow \mathrm{Eu}^{2+, *}\right)$ 
$[9,10]$. Different situation was found for the $\mathrm{Eu}$ ion in the $\mathrm{ZnS}[8]$. There, the carrier trapping proceeds via the extended excited state of the RE ion (charge transfer state [11]), which is the lowest excited state of the $\mathrm{Eu}^{2+}$ ion in the $\mathrm{ZnS}$ [8]. Thus, carrier trapping by the ionized RE ion can be followed by either Eu core emission or by the so-called localized bound exciton emission [11]. The relevant importance of these two recombination processes is analyzed in this paper.

\section{Results and discussion}

The CaS and SrS samples studied were grown by the atomic layer epitaxy (ALE) method [12] and were doped with Eu to 0.14 w\% (weight\%) (CaS), 0.2 w\% (SrS) and with Ce to 5.8 w\% (SrS) level. Photoluminescence (PL) spectrum was excited with the Inova 200 argon laser, dispersed with the GDM 1000 double grating monochromator, and was detected with the S1 EMI photomultiplier and the Stanford Research SR530 lock-in amplifier. The samples were mounted in Leybold close cycle cryogenic refrigerator.

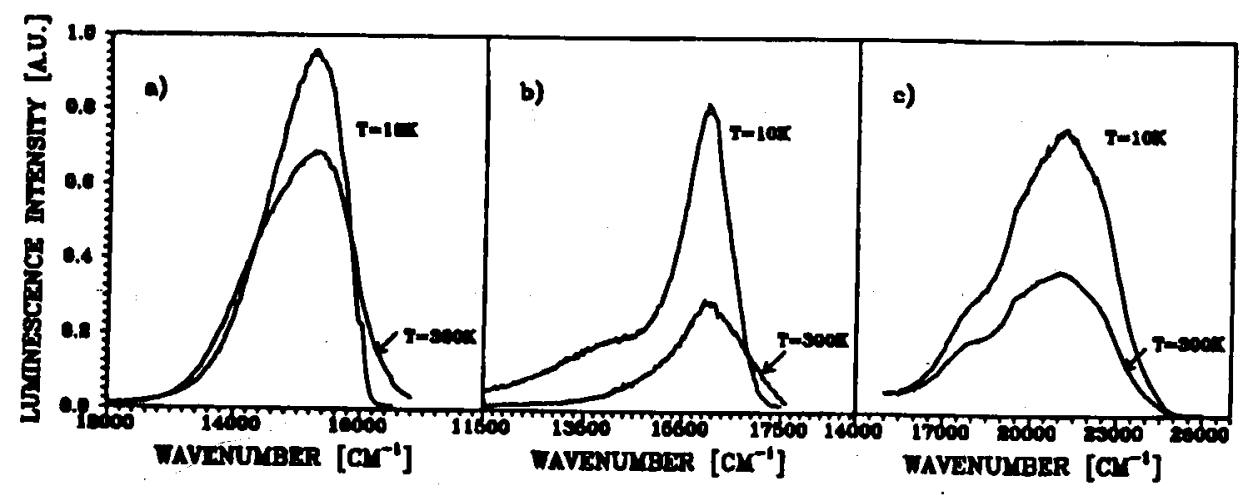

Fig. 1. The photoluminescence spectra of the ALE grown CaS:Eu (a), SrS:Eu (b) and SrS:Ce (c) samples measured at $10 \mathrm{~K}$ and $300 \mathrm{~K}$. We tentatively assign two-emission bands observed in the SrS:Eu sample to the $4 f^{6} 5 d^{1} \rightarrow 4 f^{7}$ transition of the Eu ion in two different lattice sites.

The measured PL spectrum, shown in Fig. 1a-c, consists of broad red (CaS, $\mathrm{SrS}: \mathrm{Eu}$ ) and blue (SrS:Ce) emission lines. Large width of these PL spectra is caused by inhomogeneous structure of thin films. The PL spectrum of the SrS:Eu sample shows a two-band structure, which was not observed for the samples with the larger Eu concentration [13].

Figure 2a, b shows the temperature dependence for Eu doped $\mathrm{CaS}$ and $\mathrm{SrS}$ samples. In both cases the resulting RE emission can be thermally deactivated. The observed decrease in the PL intensity with increasing temperature can be described by [9]

$$
I(T)=\frac{I(0)}{1+A \exp (-\Delta E / k T)} .
$$




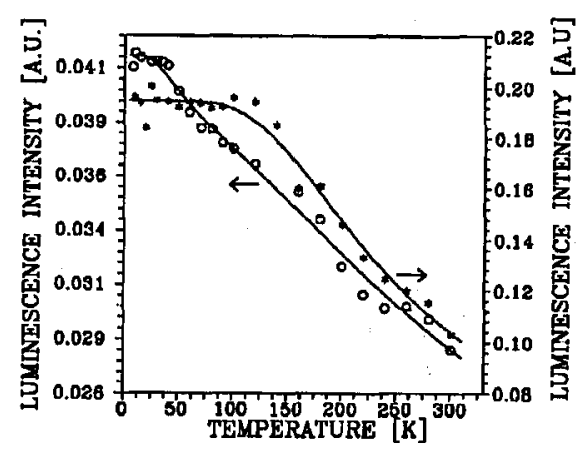

Fig. 2. The temperature dependence of the $\mathrm{Eu}^{2+} 4 f^{6} 5 d^{1} \rightarrow 4 f^{7}$ emission in the CaS (o) and $\operatorname{SrS}(*)$. In the latter case the higher energy part of the emission spectrum is analyzed. The solid line presents the fit of the formula (1) (SrS:Eu) and formula (2) (CaS:Eu) to the experimental data. The fitting parameters are given in the text.

The above formula well describes the temperature dependence of the SrS:Ce and SrS:Eu (higher energy PL) emissions with $\Delta E=6 \mathrm{meV}$ (SrS:Ce) and $\Delta E=$ $52 \mathrm{meV}$ (SrS:Eu). However, the CaS:Eu and SrS:Eu (lower energy band) data are better described by the two-exponential dependence

$$
I(T)=\frac{I(0)}{1+A_{1} \exp \left(-\Delta E_{1} / k T\right)+A_{2} \exp \left(-\Delta E_{2} / k T\right)}
$$

with $\Delta E_{1}$ and $\Delta E_{2}$ equal to $9 \mathrm{meV}$ and $50 \mathrm{meV}$ for $\mathrm{Eu}$ ion in $\mathrm{CaS}$, and $12 \mathrm{meV}$ and $90 \mathrm{meV}$ for $\mathrm{SrS}: \mathrm{Eu}$, respectively.

The excitation energy of Eu emission was selected within the broad photoionization bands. Thus, Eu ions are ionized by the incident light and the subsequent carrier trapping must occur for the $\mathrm{Eu}^{2+}$ excitation. When carrier is trapped, it can be thermally ionized back to the continuum states, which deactivates PL. The PL changes can be described by the formula (1). However, we found that for $\mathrm{CaS}: \mathrm{Eu}$ and for lower energy band in SrS:Eu the formula (2) better describes the experimental results. This suggests that in these two cases the carrier trapping is a two-step process. Carrier is first trapped to some intermediate state from which it can relax to the $4 f^{6} 5 d^{1}$ state of the $\mathrm{Eu}^{2+}$ ion or can be thermally excited to the continuum states. The nature of intermediate state is not known. However, by analogy to our previous results for $\mathrm{Eu}$ and $\mathrm{Yb}$ doped $\mathrm{ZnS}[7,8]$, we propose that it is the charge transfer state of the Eu ion. Following this idea, we tentatively ascribe the larger of the two deactivation energies to the energy distance between the $4 f^{6} 5 d^{1}$ excited state of the $\mathrm{Eu}^{2+}$ ion and the edge of the $\mathrm{CaS}, \mathrm{SrS}$ conduction band. The obtained energies depend on the Eu concentration in the sample [9, 13]. We ascribe the lower energy to the binding energy of the delocalized carrier bound at the Eu ion, i.e., to the binding energy of the charge transfer state of the ion.

The first experimental results for $\mathrm{Ce}$ ion in the $\mathrm{SrS}$ indicate that $\mathrm{Ce}^{3+}$ emission in this material is also thermally deactivated. The similarity between $\Delta E$ and $\Delta E_{1}$ deactivation energies observed in the experiment suggests that the $\Delta E$ 
energy describes the carrier emission from the charge transfer state of the Ce ion in SrS.

\section{References}

[1] M. Leskelä, L. Nïnistö, Mater. Chem. Phys. 31, 7 (1992).

[2] S. Tanaka, J. Lumin. 40/41, 20 (1988).

[3] J.W. Allen, in: Electroluminescence, Springer Proc. in Phys., Vol. 38, Eds. S. Shionoya, H. Kobayashi, Springer, Berlin 1989, p. 10.

[4] K. Świątek, A. Suchocki, M. Godlewski, Appl. Phys. Lett. 56, 195 (1990).

[5] M. Godlewski, K. Świątek, A. Suchocki, J.M. Langer, J. Lumin. 48/49, 23 (1991).

[6] M. Godlewski, K.Świątek, J. Cryst. Growth 117, 634 (1992).

[7] H. Przybylińska, K. Świątek, A. Stąpor, A. Suchocki, M. Godlewski, Phys. Rev. B 40, 1748 (1989).

[8] K. Świątek, M. Godlewski, D. Hommel, Phys. Rev. B 42, 3628 (1990).

[9] M. Ando, Y.A. Ono, J. Cryst. Growth 117, 969 (1992).

[10] K. Świątek, M. Godlewski, D. Hommel, M. Leskelä, L. Niinistö, E. Nykanen, P. Soininen, M. Tiitta, Acta Polytechn. Scand. Ser. Appl. Phys. 170, 237 (1990).

[11] M. Godlewski, Acta Phys. Pol. A 84, 849 (1993).

[12] M. Tammenmaa, H. Antson, M. Asplund, L. Hiltunen, M. Leskelä, L. Niinistö, E. Ristolainen, J. Cryst. Growth 84, 151 (1987).

[13] K. Świątek, unpublished results. 